\title{
Surgery and Anesthesia Exposure Is Not a Risk Factor for Cognitive Impairment After Major Noncardiac Surgery and Critical IIIness
}

\author{
Christopher G. Hughes, MD*, Mayur B. Patel, MD, MPH ${ }^{\dagger}$, James C. Jackson, Psy.D ${ }^{\ddagger}$, \\ Timothy D. Girard, MD, MSCI§, Sunil K. Geevarghese, MDף, Brett C. Norman, MD, MPH", \\ Jennifer L.Thompson, MPH ${ }^{\star \star}$, Rameela Chandrasekhar, PhD ${ }^{\star \star}$, Nathan E. Brummel, MD, \\ MSCI $^{\dagger \dagger}$, Addison K. May, MD $\ddagger \ddagger$, Mark R. Elstad, MD§§, Mitzi L. Wasserstein, MD ${ }^{1 \uparrow, ~ R i c h a r d ~}$ \\ B. Goodman, MD ${ }^{\|\| \prime}$, Karel G. Moons, PhD ${ }^{\star \star \star}$, Robert S. Dittus, MD, MPH§, E. Wesley Ely, MD, \\ MPH§, Pratik P. Pandharipande, MD, MSCI't†, and for the MIND-ICU, BRAIN-ICU \\ investigators
}

*Department of Anesthesiology, Division of Anesthesiology Critical Care Medicine and Center for Health Services Research, Vanderbilt University Medical Center, Nashville Veterans Affairs Medical Center, Tennessee Valley Healthcare System, Nashville, Tennessee tSection of Surgical Sciences, Departments of Surgery, Neurosurgery and Hearing and Speech Sciences, Division of Trauma and, Surgical Critical Care, and Emergency General Surgery, Vanderbilt Brain Institute, Center for Health Services Research, Vanderbilt University Medical Center, Nashville Veterans Affairs Medical Center, Tennessee Valley Healthcare System, Nashville, Tennessee ${ }^{\ddagger}$ Department of Medicine, Division of Pulmonary and Critical Care Medicine and Center for Health Services Research, Vanderbilt University Medical Center, Research Service, Nashville Veterans Affairs Medical Center, Tennessee Valley Healthcare System, Nashville, Tennessee §Department of Medicine, Division of Pulmonary and Critical Care Medicine and Center for Health Services Research, Vanderbilt University Medical Center, Geriatric Research, Education and Clinical Center Service, Nashville Veterans Affairs Medical Center, Tennessee Valley Healthcare System, Nashville, Tennessee 'Depart-Department of Surgery, Radiology, and Radiological Sciences, Vanderbilt University Medical Center, Nashville Veterans Affairs Medical Center, Tennessee Valley Healthcare System, Nashville, Tennessee "Department of Medicine, Division of Pulmonary and Critical Care Medicine, Vanderbilt University Medical Center, Nashville, Tennessee ${ }^{* *}$ Department of Biostatistics, Vanderbilt University School of Medicine, Nashville, Tennessee ttDepartment of Medicine, Division of Pulmonary and Critical Care Medicine, Vanderbilt University School of

Reprints: Christopher G. Hughes, MD, 1211 21st Ave. South, 526 MAB, Nashville, TN 37212. christopher.hughes@ vanderbilt.edu. MIND-ICU, BRAIN-ICU investigators: Delirium and Dementia in Veterans Surviving ICU Care (MIND-ICU) and Bringing to Light the Risk Factors and Incidence of Neuropsychological Dysfunction in ICU Survivors (BRAIN-ICU).

CGH has received honoraria from Orion Pharma. TDG has received honoraria from Hospira, Inc. AKM has received research grants from GlaxoSmithKline, BHR Pharma, Sanofi-Aventis, Cubist Pharmaceuticals, and Fresenius Kabi. EWE has received honoraria from Abbott Laboratories, Hospira, Inc., and Orion Corporation and research grants from Abbott Laboratories and Hospira, Inc. PPP has received research grants from Hospira, Inc. MBP, JCJ, SKG, BCN, JLT, RC, NEB, MRE, MLW, RBG, KGM, and RSD declare no competing interests.

The authors declare no conflict of interests.

Supplemental digital content is available for this article. Direct URL citations appear in the printed text and are provided in the HTML and PDF versions of this article on the journal's Web site (www.annalsofsurgery.com). 
Medicine, Nashville, Tennessee $¥$ Section of Surgical Sciences, Department of Surgery, Division of Trauma and Surgical Critical Care, and Emergency General Surgery, Vanderbilt University Medical Center, Nashville, Tennessee §§Department of Internal Medicine, Division of Pulmonary and Critical Care Medicine, University of Utah School of Medicine, George E. Wahlen Department of Veterans Affairs Medical Center, Veterans Administration Salt Lake City Health Care System, Salt Lake City, Utah "George E. Wahlen Department of Veterans Affairs Medical Center, Veterans Administration Salt Lake City Health Care System, Salt Lake City, Utah "'IDepartment of Internal Medicine, Division of Pulmonary and Critical Care Medicine, University of Washington School of Medicine, Seattle Division, Veterans Affairs Puget Sound Health Care System, Seattle, Washington ${ }^{* *}$ Clinical Epidemiology, Julius Center for Health Sciences and Primary Care, University Medical Center, Utrecht, the Netherlands ${ }^{\dagger+t}$ Departments of Anesthesiology and Surgery, Division of Anesthesiology Critical Care Medicine, Vanderbilt University Medical Center, Nashville Veterans Affairs Medical Center, Tennessee Valley Healthcare System, Nashville, Tennessee

\section{Abstract}

Objective-The aim of this study was to determine whether surgery and anesthesia exposure is an independent risk factor for cognitive impairment after major noncardiac surgery associated with critical illness.

Summary of Background Data-Postoperative cognitive impairment is a prevalent individual and public health problem. Data are inconclusive as to whether this impairment is attributable to surgery and anesthesia exposure versus patients' baseline factors and hospital course.

Methods-In a multicenter prospective cohort study, we enrolled ICU patients with major noncardiac surgery during hospital admission and with nonsurgical medical illness. At 3 and 12 months, we assessed survivors' global cognitive function with the Repeatable Battery for the Assessment of Neuropsychological Status and executive function with the Trail Making Test, Part B. We performed multivariable linear regression to study the independent association of surgery/ anesthesia exposure with cognitive outcomes, adjusting initially for baseline covariates and subsequently for in-hospital covariates.

Results-We enrolled 1040 patients, 402 (39\%) with surgery/anesthesia exposure. Median global cognition scores were similar in patients with surgery/anesthesia exposure compared with those without exposure at 3 months (79 vs 80 ) and 12 months (82 vs 82 ). Median executive function scores were also similar at 3 months ( 41 vs 40 ) and 12 months (43 vs 42 ). Surgery/ anesthesia exposure was not associated with worse global cognition or executive function at 3 or 12 months in models incorporating baseline or in-hospital covariates $(P>0.2)$. Higher baseline education level was associated with better global cognition at 3 and 12 months $(P<0.001)$, and longer in-hospital delirium duration was associated with worse global cognition $(P<0.02)$ and executive function $(P<0.01)$ at 3 and 12 months.

Conclusions-Cognitive impairment after major noncardiac surgery and critical illness is not associated with the surgery and anesthesia exposure but is predicted by baseline education level and in-hospital delirium. 


\section{Keywords}

anesthesia; cognition; delirium; executive function; surgery

Cognitive impairment after surgery and anesthesia—termed postoperative cognitive dysfunction (POCD) - is an individual and public health problem associated with increased mortality and decreased employment. ${ }^{1-8}$ Nevertheless, the characteristics and the magnitude of this cognitive impairment have been difficult to describe given the varied neuropsychological tests used in prior studies. Moreover, the lack of comparative cognitive outcomes data from patients who undergo surgery compared with those who do not, as well as to those with disease states such as traumatic brain injury (TBI), vascular dementia, and Alzheimer disease (AD), prevents placing this cognitive impairment after surgery and anesthesia into clinical context.

Age, education level, and pre-existing cognitive deficits have been shown to be associated with cognitive impairment after anesthesia and surgery, ${ }^{6,9,10}$ and some studies have shown that surgical and anesthetic factors may also play a role. ${ }^{6,11,12}$ Additional studies, however, have called this into question. For instance, cognitive outcomes are similar after invasive surgery requiring general anesthesia versus those performed under regional anesthesia ${ }^{8,13,14}$ or even percutaneous procedures requiring moderate sedation. ${ }^{15-17}$ Cognitive outcomes after coronary artery bypass grafting are similar to or improved from baseline ${ }^{18}$ and similar to those with coronary artery disease managed medically. ${ }^{19}$ Furthermore, reducing depth of anesthesia does not affect the incidence of cognitive impairment after surgery. ${ }^{20}$ Thus, it is unclear whether exposure to surgery and anesthesia plays an independent role or whether this cognitive impairment is secondary to preexisting patient comorbidities or events during the hospital course. Most studies to date have focused on cardiac or elective joint replacement surgeries, have not utilized medical illness comparator groups, or have not examined significant postoperative factors such as delirium, which was recently shown to be associated with worse cognitive impairment up to a year after cardiac surgery. ${ }^{21}$

We hypothesized that exposure to surgery and anesthesia would not be associated with cognitive impairment in patients undergoing major noncardiac surgery. We performed a prospective cohort study of patients admitted to the intensive care unit (ICU) who underwent major noncardiac surgical procedures during their hospital admission and patients admitted to the ICU with nonsurgical medical illness, evaluating them for cognitive impairment 3 and 12 months after discharge. We tested the independent associations of exposure to surgery and anesthesia (yes/no) and increasing level of that exposure with cognitive impairment, adjusting for baseline and in-hospital factors.

\section{METHODS}

\section{Study Design and Population}

This prospective cohort study is a combined investigation of the MIND-ICU Study:

Delirium and Dementia in Veterans Surviving ICU Care study (NCT00400062) conducted at the Tennessee Valley Healthcare System (Nashville, TN), George E. Wahlen Department of 
VA Medical Center in VA Salt Lake City Health Care System (Salt Lake City, UT), and Seattle Division of the VA Puget Sound Health Care System (Seattle, WA) and the parallel (identical protocol) Bringing to Light the Risk Factors and Incidence of Neuropsychological Dysfunction in ICU Survivors (BRAIN-ICU) ${ }^{22}$ study (NCT00392795) conducted at Vanderbilt University Medical Center and Saint Thomas Hospital (both Nashville, TN). Results from the BRAIN-ICU study have been previously reported. ${ }^{22,23}$ The hypotheses tested in this larger combined cohort are original and have not been previously published.

The study protocol was approved by each institution's review board. We enrolled adult patients in respiratory failure or shock, including after major surgical procedures and nonsurgical medical illnesses, as previously described. ${ }^{22,23}$ We excluded patients with recent critical illness, moribund state, inability to perform delirium assessments, high likelihood of preexisting cognitive deficits, admission for stroke or neurological surgery, limited outpatient follow-up opportunity, and inability to obtain informed consent. See Supplemental Digital Content, http://links.lww.com/SLA/B51 for further details.

\section{Exposures}

Our primary independent variable was surgery requiring general anesthesia in an operating room, which was determined by daily review of the medical record and prospectively collected. As general anesthesia and surgery occur together, we labeled the independent variable as "surgery/anesthesia exposure." Patients who had surgery with general anesthesia at any point during their current hospital admission before enrollment or within any time between enrollment and 30 days postenrollment were considered to have surgery/anesthesia exposure, irrespective of whether they were admitted to a medical or surgical ICU. In a subset of patients with surgery/anesthesia exposure, more robust data were obtained, including number of surgeries (total trips to the operating room while in the hospital), emergent surgery (yes/no), and total duration of anesthesia (sum of individual anesthetic exposures if more than 1) in order to test the relationship between increasing surgery/ anesthesia exposure and outcomes.

\section{Outcomes}

At 3 and 12 months after hospital discharge, trained psychology professionals who were blinded to hospital course and surgery status assessed patients' global cognitive function with the Repeatable Battery for the Assessment of Neuropsychological Status (RBANS), ${ }^{24}$ a validated neuropsychometric battery that measures domains of memory (immediate and delayed), visuospatial construction, language, and attention. The RBANS has a mean (standard deviation) population age-adjusted score of $100 \pm 15$ with lower scores indicating worse global cognitive function. The RBANS has also been validated in patients with $\mathrm{TBI}^{25}$ and mild $\mathrm{AD},{ }^{24}$ with mean scores of 78 and 70 in these populations, respectively. The RBANS domain scores have further been validated to differentiate between cortical dementias (such as AD) and subcortical dementias (vascular dementias, Huntington dementia), acknowledging the potential limitations of such a categorization approach. ${ }^{24,26,27}$ A single cortical-subcortical deviation score is calculated by subtracting the mean of the delayed memory index and the language index from the mean of the attention index and the visuospatial constructional index. ${ }^{24,26,27}$ Patients with cognitive impairment and a score $>0$ 
are considered to have cortical dementias, and those patients with a score $<0$ have subcortical dementias. In addition to global cognitive function, executive function was assessed with the Trail Making Test, Part B (Trails B), ${ }^{28}$ a validated tool that examines cognitive flexibility and set shifting. The Trails B has an age-, sex-, and education- adjusted mean score of $50 \pm 10$ with lower scores indicating worse executive function.

\section{Confounders}

We collected demographic data upon enrollment and hospital course data from admission to 30 days after enrollment. We chose a priori potential confounders of the association between surgery/ anesthesia exposure and cognitive impairment on the basis of previous research and clinical judgment. Baseline confounders included age, years of education, comorbid disease burden with the Charlson comorbidity index, cerebrovascular disease with the Framingham stroke risk score, and pre-existing cognitive deficit with the Short Form Informant Questionnaire on Cognitive Decline in the Elderly (IQCODE-SF ${ }^{29}$ score. The IQCODE-SF has a sensitivity $>0.80$ to identify mild cognitive impairment ${ }^{30,31}$ and $>0.90$ to identify adults at risk of dementia, including in the hospital. ${ }^{32}$ In-hospital confounders included duration of coma, duration of delirium, duration of severe sepsis, duration of hypoxemia, severity of illness with the Sequential Organ Failure Assessment Score, analgesic exposure, sedative exposure, and antipsychotic exposure. See Supplemental Digital Content, http:// links.lww.com/SLA/B51 for further details.

\section{Statistical Analysis}

We used multivariable linear regression to determine whether surgery/anesthesia exposure (yes/no) was independently associated with the primary outcome variables (RBANS and Trails B scores at 3 and 12 months). Among our entire cohort, we first evaluated surgery/ anesthesia exposure (yes/no) on cognitive outcomes adjusting for baseline covariates alone. We then performed linear regression analysis that included in-hospital covariates in addition to baseline covariates to determine the predictors of cognitive impairment. We included predefined interactions between surgery/anesthesia exposure and education and duration of delirium as well as an interaction between durations of delirium and coma. Finally, in the subset of surgical patients with robust details regarding their surgery/anesthesia exposure, we performed multivariable regression to examine whether having a higher number of surgeries, emergent surgery, and/or a longer total duration of anesthesia was associated with cognitive outcomes, accounting for baseline covariates.

In all models, continuous variables were modeled with the use of restricted cubic splines to allow for nonlinear associations (with the exception of dexmedetomidine and haloperidol doses; due to infrequent use of these medications, there were too few unique doses to allow splines). In order to reduce bias from missing data, we used multiple imputation in our analyses to include in our analyses any patient who had some missing covariates or who had at least partially completed cognitive assessments. No imputations were performed for completely missing cognitive assessments. Overall, imputed data were utilized in $<7 \%$ of subjects at 3 months and $<5 \%$ of subjects at 12 months (see Supplemental Digital Content, http://links.lww.com/SLA/B51). We used R version 3.1.2 (https://www.r-project.org/) for all 
statistical analyses and considered $P<0.05$ as statistical significance for independent variables.

\section{RESULTS}

Between March 2007 and May 2010, we enrolled 1046 patients (Supplemental Figure 1, http://links.lww.com/SLA/B51); 6 patients withdrew consent and requested data destruction. Thus, we included 1040 patients with a median (interquartile range) age of 62 (53 to 72) years and a high severity of illness (Table 1), 402 (39\%) with surgery/anesthesia exposure, and 638 without exposure. After accounting for death, loss to follow-up, and withdrawal, 534 patients had cognitive testing at 3 and/or 12 months (Supplemental Figure 1, http:// links.lww.com/SLA/B51), 219 (41\%) with surgery/anesthesia exposure, and 315 without exposure.

Median RBANS global cognition scores were similar among patients with surgery/ anesthesia exposure and among those without exposure $[79(71,86)$ vs $80(70,87)$ at 3 months; $82(72,90)$ vs $82(73,90)$ at 12 months], approximately 1.5 standard deviations below adjusted population mean, a level indicative of significant cognitive impairment (Fig. 1). Among the patients with surgery/anesthesia exposure, $44 \%$ and $35 \%$ earned scores below those typical of patients with TBI at 3 and 12 months, respectively (39\% and 35\% in the group without exposure). Furthermore, 24\% and 23\% scored below those typical of patients with $\mathrm{AD}$ at 3 and 12 months (25\% and 21\% in those without exposure). Among all patients with scores indicative of cognitive impairment ( $<78$ on RBANS; 1.5 standard deviation below population mean), cortical-subcortical deviation score distribution was similar in patients with surgery/anesthesia exposure and in those without exposure (73\% vs $74 \%$ had RBANS cortical-subcortical deviation scores $<0$, displaying a subcortical pattern as seen in vascular dementia, at 3 months; $69 \%$ vs $63 \%$ had scores indicative of a subcortical pattern at 12 months). Median Trails B executive function scores were also similar among patients with surgery/ anesthesia exposure and among those without exposure $[41(33,48)$ vs $40(33$, $48)$ at 3 months; $43(37,52)$ vs $42(35,49)$ at 12 months], approximately 1 standard deviation below adjusted population mean (Fig. 1).

In models incorporating only baseline covariates (Table 2), surgery/anesthesia exposure (yes/no) was not associated with RBANS global cognition or Trails B executive function scores at 3 or 12 months $(P>0.2)$. Pre-existing cognitive deficit as indicated by lower scores on the IQCODE-SF was associated with worse RBANS global cognition scores at 3 and 12 months $(P<0.001 ; P=0.04)$, and higher years of education was associated with better scores $(P<0.001 ; P<0.001)$. Pre-existing cognitive deficit by the IQCODE-SF was the only variable associated with lower Trails B executive function scores at 3 and 12 months ( $P$ $=0.03 ; P<0.001)$. In the subset of 233 surgical patients with additional analyses, neither higher number of surgeries, emergent surgery, nor longer total duration of general anesthesia was associated with worse RBANS global cognition or Trails B executive function scores at 3 or 12 months ( $P>0.06$; Table 3). See Supplemental Table 1, http:// links.lww.com/SLA/B51 for details on the surgical and anesthesia characteristics. 
In models incorporating baseline and in-hospital covariates (Table 4), surgery/anesthesia exposure (yes/no) remained unassociated with RBANS global cognition or Trails B executive function scores at 3 or 12 months $(P>0.3)$. At both 3 and 12 months, increasing age $(\mathrm{P}<0.001 ; \mathrm{P}=0.01)$ and longer delirium duration $(P=0.01 ; P=0.003)$ were associated with worse RBANS global cognition scores, and higher years of education was associated with better scores $(P<0.001 ; P<0.001)$. Longer delirium duration was associated with lower Trails B executive function scores at both 3 and 12 months $(P=0.01 ; P<0.001)$. We did not detect any statistically significant interactions between surgery/anesthesia exposure and education or duration of delirium.

\section{DISCUSSION}

In this multicenter prospective cohort study involving patients with surgical and nonsurgical critical illness at academic, community, and Veterans Affairs hospitals, we found that surgery/anesthesia exposure was not a risk factor for long-term global cognitive function or executive function impairment after major noncardiac surgery. In addition, increasing level of exposure as measured by number of surgeries, emergent surgery, and duration of anesthesia was not associated with worse global cognition or executive function. Cognitive impairment was highly prevalent at 3 and 12 months after hospital discharge and affected patients who were exposed to general anesthesia and surgery at rates similar to those who did not undergo a surgical procedure. This impairment was associated with pre-existing cognitive deficits and level of education; longer delirium duration was the only identified inhospital risk factor for worse cognitive outcomes in our cohort. Over one-third of patients and approximately one quarter of patients scored at levels comparable to or worse than individuals with TBI and AD, respectively. The impairment we observed did not appear to be primarily of an Alzheimer type, as reflected in the subcortical (eg, vascular dementia) rather than cortical nature of deficits.

Our results complement and build upon the results of earlier cohort studies that have identified significant cognitive impairment in hospitalized and postsurgical patients, 2,4,6,7,21,22,33,34 but our work has unique characteristics. First, we enrolled surgical and medical patients from civilian and veteran populations from across the Unites States and had high follow-up rates for assessments after discharge, making our cohort generalizable to a broad group of patients and allowing the patients without surgery/anesthesia exposure to serve as a medical comparator group often lacking in previous studies. Second, we obtained detailed hospital-course data and performed daily acute brain dysfunction assessments, allowing us to examine in-hospital factors that are almost never included in prior beforeafter studies of perioperative cognitive trajectory. Third, we performed extensive inperson assessments with the validated RBANS battery and Trails B test and demonstrated that a significant number of surgical patients have levels of impairment similar to or worse than $\mathrm{TBI}$ or $\mathrm{AD}$. The impairment in our patients was predominantly subcortical in nature, similar to vascular dementia as opposed to AD. For instance, our patients were relatively more likely to demonstrate deficits in domains of attention and visuo-spatial construction than in domains of language and delayed memory, although neuropsychological deficits were common across virtually all domains. Many previous studies assessed cognition with abbreviated screening tools or failed to compare impairment seen after surgery with those 
seen in reference populations such as TBI or AD. Our findings, therefore, provide a better appreciation of the range and magnitude of deficits and the possible societal implications that these may have.

Our results support the increasing evidence that preoperative and postoperative factors are the most important determinants of postoperative cognitive trajectory, not the operative event itself. ${ }^{10,21,35,36}$ By initially analyzing only surgery/anesthesia exposure and baseline factors (eg, age, education level, pre-existing deficits, stroke risk) in this mixed population, we were able to characterize better the associations between preexisting comorbidities and cognitive outcomes distinctly from the subsequent events of the hospital course. We then investigated the role of the hospital course on cognitive outcomes and examined a large number of potential clinical and pathophysiologic risk factors, including duration of delirium and coma, sepsis, hypoxemia, severity of illness, and sedative exposure, which are often inadequately characterized or not studied due to lack of robust data collection and statistical power. In both analyses, we did not find a significant independent association between surgery/ anesthesia exposure and cognitive outcomes at 3 or 12 months. Furthermore, given the small effect size estimates and confidence intervals associated with surgery/anesthesia exposure, it is unlikely that there would be more than a 2.5 unit decrease in the RBANS based on the largest estimated upper confidence limit for the effect. This extent of change is not clinically significant, ${ }^{37}$ and we can safely rule out an effect of that magnitude, if any at all, from surgery/ anesthesia exposure.

Although we cannot exclude the possibility that some mild degree of dysfunction might occur from the surgery/anesthesia exposure, any contribution from this exposure, if present, would be far less than the contribution of baseline and in-hospital factors for this critically ill cohort. These results are consistent with a recent study of over 8000 twins that found no significant association of major surgery and anesthesia with long-term cognitive dysfunction. ${ }^{36}$ Importantly, our study examined a void in the current literature-high-risk major noncardiac surgical patients with extensively analyzed postoperative courses—but our findings may not be applicable to noncritically ill patients exposed to lower acuity surgery or regional anesthesia techniques with less resulting cognitive impairment. Prior studies, however, support the lack of association in these populations as well. ${ }^{8,13-15,17,20}$ In addition, we did not find an association between surgery/anesthesia exposure and outcomes in our analyses when including baseline covariates only, thus excluding critical illness factors.

Of the risk factors examined, longer duration of delirium was the only potentially modifiable in-hospital factor that was associated with worse global cognitive function and executive function at 3 or 12 months. This association is consistent with recent results in both surgical and medical patients demonstrating that delirium during a hospitalization may portend worse long-term outcomes, including greater risk of cognitive, physical, and social functioning decline and need for institutionalization. ${ }^{38-40}$ For example, cardiac surgery patients with delirium had a larger decrease in cognitive function measured by the Mini-Mental State Examination at both 1 month and 12 months than those without delirium ${ }^{21}$; furthermore, a higher percentage of patients with delirium did not return to baseline function than those without delirium at 6 and 12 months. ${ }^{21}$ This study also extends our BRAIN-ICU findings demonstrating that delirium was associated with worse cognitive testing scores 3 and 12 
months after critical illness. ${ }^{22}$ Thus, delirium, as a manifestation of acute brain organ dysfunction in patients with surgical or medical illness, portends significant chronic brain dysfunction.

We found that higher education level was independently associated with better global cognitive function at 3 and 12 months, whereas pre-existing cognitive deficit was associated with worse global cognitive function. This is similar to findings in other disease states such as $\mathrm{TBI}$ and $\mathrm{AD}^{41,42}$ and supports the premise that cognitive reserve is protective of longterm impairment after acute insult. Patients with higher cognitive reserve likely possess increased capacity to maintain normal functioning in response to stress (eg, surgical or medical illness) and are, therefore, at a lower risk for subsequent impairment. Therapeutic approaches for improving cognitive reserve may present opportunities for reducing cognitive impairment after acute stressors, particularly elective surgeries with time available for prehabilitation. ${ }^{43,44}$

Our study has many strengths as outlined above but has a few limitations that warrant discussion. We were unable to account for depth of anesthesia and extent of surgical tissue injury. We were also unable to account for past surgery/anesthesia exposure or for prior delirium episodes related to past hospitalizations that could have altered cognitive trajectories. Despite being unable to perform extensive cognitive testing before hospitalization, we did obtain information on patients' pre-existing cognitive functioning. The IQCODE-SF, ${ }^{29}$ which has high sensitivity to identify mild cognitive impairment and dementia, ${ }^{30-32}$ was used to account for baseline cognitive deficits and was the same in patients with surgery/anesthesia exposure and in those without exposure. Furthermore, other demographic characteristics associated with baseline cognitive status (eg, age, education level, socioeconomic status, frailty, functional activity level, comorbidities) were collected and were also similar between groups. These findings indicate that both groups had similar pre-admission cognitive functioning. Delirium assessments were not coordinated with interruption of sedation; patients considered delirious in our cohort may have included patients with rapidly reversible sedation-related delirium (previously reported to affect $12 \%$ of patients in a study of 102 patients and not associated with worse outcomes), ${ }^{45}$ potentially biasing our delirium results toward the null hypothesis. Finally, observational studies cannot prove causation and are subject to unmeasured confounders; however, we were able to adjust for a large number of confounders due to the size of the cohort, including baseline and inhospital factors.

In conclusion, exposure to surgery and general anesthesia is not a significant risk factor for long-term cognitive impairment after major noncardiac surgery associated with critical illness. Cognitive impairment is prevalent at 3 and 12 months after hospital discharge and primarily associated with baseline education level and longer duration of delirium in the hospital.

\section{Supplementary Material}

Refer to Web version on PubMed Central for supplementary material. 


\section{Acknowledgments}

We acknowledge our research coordinators Leanne Boehm, MSN, RN, ACNS-BC; Brenda Truman Pun, RN, MSN; Joyce Okahashi, RN; and Cayce Strength, RSN, BSN who performed project management, data acquisition, and data management. We acknowledge our neuropsychology staff Amy Kiehl, MA, LPC-MHSP who performed neuropsychological outcome testing.

CGH is supported by a Foundation for Anesthesia Education and Research (Rochester, MN) Mentored Research Training Grant, American Geriatrics Society Jahnigen Career Development Award, and National Institutes of Health HL111111, R03AG045085 (Bethesda, MD). MBP is supported by the Vanderbilt Faculty Research Scholars Program and National Institutes of Health HL111111 (Bethesda, MD). TDG is supported by the National Institutes of Health AG034257, AG035117 (Bethesda, MD). NEB is supported by the National Institutes of Health KL2 TR00046 and R03AG040549 (Bethesda, MD) and by the Vanderbilt Clinical and Translational Scholars program. KGM is supported by the Netherlands Organization for Scientific Research (project 9120.8004 and 918.10.615). TDG, RSD, and EWE are supported by the Veterans Affairs Tennessee Valley Geriatric Research, Education and Clinical Center (Nashville, TN). RSD, EWE, and PPP are supported by the VA Clinical Science Research and Development Service (Washington, DC) and the National Institutes of Health AG027472, AG035117, HL111111 (Bethesda, MD). This project was supported by National Institutes of Health AG027472 and UL1 RR024975 (Bethesda, MD) and a Merit Review Grant from Department of Veterans Affairs (Washington, DC). We used REDCap, a secure online database, supported in part by the National Institutes of Health TR000445. The funders of the study had no role in study design, data collection, data analysis, data interpretation, or writing of the report.

CGH, MBP, JCJ, TDG, NEB, KGM, EWE, and PPP developed the study concept and design. All authors were involved in data acquisition and interpretation. CGH, MBP, JCJ, EWE, and PPP had primary responsibility for writing of the manuscript with critical revision and final approval performed by all authors. JLTand RC completed all data analyses. CGH, EWE, and PPP obtained funding for the study, and CGH, JCJ, TDG, EWE, and PPP supervised the conduct of the study. CGH and PPP had full access to all of the data in the study, take responsibility for the integrity of the data and the accuracy of the data analysis, and had final responsibility for the decision to submit for publication.

\section{References}

1. Steinmetz J, Christensen KB, Lund T, et al. Long-term consequences of postoperative cognitive dysfunction. Anesthesiology. 2009; 110:548-555. [PubMed: 19225398]

2. Newman MF, Kirchner JL, Phillips-Bute B, et al. Longitudinal assessment of neurocognitive function after coronary-artery bypass surgery. N Engl J Med. 2001; 344:395-402. [PubMed: 11172175]

3. McDonagh DL, Mathew JP, White WD, et al. Cognitive function after major noncardiac surgery, apolipoprotein E4 genotype, and biomarkers of brain injury. Anesthesiology. 2010; 112:852-859. [PubMed: 20216394]

4. van Dijk D, Spoor M, Hijman R, et al. Cognitive and cardiac outcomes 5 years after off-pump vs onpump coronary artery bypass graft surgery. JAMA. 2007; 297:701-708. [PubMed: 17312289]

5. Ottens TH, Dieleman JM, Sauer AM, et al. Effects of dexamethasone on cognitive decline after cardiac surgery: a randomized clinical trial. Anesthesiology. 2014; 121:492-500. [PubMed: 25225745]

6. Moller JT, Cluitmans P, Rasmussen LS, et al. Long-term postoperative cognitive dysfunction in the elderly ISPOCD1 study. ISPOCD investigators. International Study of Post-Operative Cognitive Dysfunction. Lancet. 1998; 351:857-861. [PubMed: 9525362]

7. Quinlan N, Rudolph JL. Postoperative delirium and functional decline after noncardiac surgery. J Am Geriatr Soc. 2011; 59:S301-S304. [PubMed: 22091577]

8. Williams-Russo P, Sharrock NE, Mattis S, et al. Cognitive effects after epidural vs general anesthesia in older adults. A randomized trial. JAMA. 1995; 274:44-50. [PubMed: 7791257]

9. Monk TG, Weldon BC, Garvan CW, et al. Predictors of cognitive dysfunction after major noncardiac surgery. Anesthesiology. 2008; 108:18-30. [PubMed: 18156878]

10. Silbert B, Evered L, Scott DA, et al. Preexisting cognitive impairment is associated with postoperative cognitive dysfunction after hip joint replacement surgery. Anesthesiology. 2015; 122:1224-1234. [PubMed: 25859906] 
11. Papaioannou A, Fraidakis O, Michaloudis D, et al. The impact of the type of anaesthesia on cognitive status and delirium during the first postoperative days in elderly patients. Eur J Anaesthesiol. 2005; 22:492-499. [PubMed: 16045136]

12. Mason SE, Noel-Storr A, Ritchie CW. The impact of general and regional anesthesia on the incidence of post-operative cognitive dysfunction and postoperative delirium: a systematic review with meta-analysis. J Alzheimers Dis. 2010; 22:67-79. [PubMed: 20858956]

13. Silbert BS, Evered LA, Scott DA. Incidence of postoperative cognitive dysfunction after general or spinal anaesthesia for extracorporeal shock wave lithotripsy. Br J Anaesth. 2014; 113:784-791. [PubMed: 24972789]

14. Rasmussen LS, Johnson T, Kuipers HM, et al. Does anaesthesia cause postoperative cognitive dysfunction? A randomised study of regional versus general anaesthesia in 438 elderly patients. Acta Anaesthesiol Scand. 2003; 47:260-266. [PubMed: 12648190]

15. Evered L, Scott DA, Silbert B, et al. Postoperative cognitive dysfunction is independent of type of surgery and anesthetic. Anesth Analg. 2011; 112:1179-1185. [PubMed: 21474666]

16. Hlatky MA, Bacon C, Boothroyd D, et al. Cognitive function 5 years after randomization to coronary angioplasty or coronary artery bypass graft surgery. Circulation. 1997; 96:II-11-II-14.

17. Lal BK, Younes M, Cruz G, et al. Cognitive changes after surgery vs stenting for carotid artery stenosis. J Vasc Surg. 2011; 54:691-698. [PubMed: 21700413]

18. Shroyer AL, Grover FL, Hattler B, et al. On-pump versus off-pump coronary-artery bypass surgery. N Engl J Med. 2009; 361:1827-1837. [PubMed: 19890125]

19. Selnes OA, Grega MA, Bailey MM, et al. Cognition 6 years after surgical or medical therapy for coronary artery disease. Ann Neurol. 2008; 63:581-590. [PubMed: 18481292]

20. Radtke FM, Franck M, Lendner J, et al. Monitoring depth of anaesthesia in a randomized trial decreases the rate of postoperative delirium but not postoperative cognitive dysfunction. $\mathrm{Br} \mathrm{J}$ Anaesth. 2013; 110:i98-i105. [PubMed: 23539235]

21. Saczynski JS, Marcantonio ER, Quach L, et al. Cognitive trajectories after postoperative delirium. N Engl J Med. 2012; 367:30-39. [PubMed: 22762316]

22. Pandharipande PP, Girard TD, Jackson JC, et al. Long-term cognitive impairment after critical illness. N Engl J Med. 2013; 369:1306-1316. [PubMed: 24088092]

23. Jackson JC, Pandharipande PP, Girard TD, et al. Depression, post-traumatic stress disorder, and functional disability in survivors of critical illness in the BRAIN-ICU study: a longitudinal cohort study. Lancet Respir Med. 2014; 2:369-379. [PubMed: 24815803]

24. Randolph C, Tierney MC, Mohr E, et al. The Repeatable Battery for the Assessment of Neuropsychological Status (RBANS): preliminary clinical validity. J Clin Exp Neuropsychol. 1998; 20:310-319. [PubMed: 9845158]

25. McKay C, Casey JE, Wertheimer J, et al. Reliability and validity of the RBANS in a traumatic brain injured sample. Arch Clin Neuropsychol. 2007; 22:91-98. [PubMed: 17141467]

26. McDermott AT, DeFilippis NA. Are the indices of the RBANS sufficient for differentiating Alzheimer's disease and subcortical vascular dementia? Arch Clin Neuropsychol. 2010; 25:327_ 334. [PubMed: 20430863]

27. Beatty WW, Ryder KA, Gontkovsky ST, et al. Analyzing the subcortical dementia syndrome of Parkinson's disease using the RBANS. Arch Clin Neuropsychol. 2003; 18:509-520. [PubMed: 14591446]

28. Reitan, R., Wolfson, D. The Halstead Reitan Neuropsychological Test Battery. Tuscon, AZ: Neuropsychology Press; 1985.

29. Jorm AF. A short form of the Informant Questionnaire on Cognitive Decline in the Elderly (IQCODE): development and cross-validation. Psychol Med. 1994; 24:145-153. [PubMed: 8208879]

30. Isella V, Villa L, Russo A, et al. Discriminative and predictive power of an informant report in mild cognitive impairment. J Neurol Neurosurg Psychiatry. 2006; 77:166-171. [PubMed: 16421116]

31. Li F, Jia XF, Jia J. The Informant Questionnaire on Cognitive Decline in the Elderly individuals in screening mild cognitive impairment with or without functional impairment. J Geriatr Psychiatry Neurol. 2012; 25:227-232. [PubMed: 23172761] 
32. Harrison JK, Fearon P, Noel-Storr AH, et al. Informant Questionnaire on Cognitive Decline in the Elderly (IQCODE) for the diagnosis of dementia within a secondary care setting. Cochrane Database Syst Rev. 2015; 3:CD010772.

33. Iwashyna TJ, Ely EW, Smith DM, et al. Long-term cognitive impairment and functional disability among survivors of severe sepsis. JAMA. 2010; 304:1787-1794. [PubMed: 20978258]

34. Ehlenbach WJ, Hough CL, Crane PK, et al. Association between acute care and critical illness hospitalization and cognitive function in older adults. JAMA. 2010; 303:763-770. [PubMed: 20179286]

35. Avidan MS, Searleman AC, Storandt M, et al. Long-term cognitive decline in older subjects was not attributable to noncardiac surgery or major illness. Anesthesiology. 2009; 111:964-970. [PubMed: 19786858]

36. Dokkedal U, Hansen TG, Rasmussen LS, et al. Cognitive functioning after surgery in middle-aged and elderly Danish twins. Anesthesiology. 2016; 124:312-321. [PubMed: 26785430]

37. Harvey PD. Clinical applications of neuropsychological assessment. Dialogues Clin Neurosci. 2012; 14:91-99. [PubMed: 22577308]

38. Witlox J, Eurelings LS, de Jonghe JF, et al. Delirium in elderly patients and the risk of postdischarge mortality, institutionalization, and dementia: a metaanalysis. JAMA. 2010; 304:443451. [PubMed: 20664045]

39. Abelha FJ, Luis C, Veiga D, et al. Outcome and quality of life in patients with postoperative delirium during an ICU stay following major surgery. Crit Care. 2013; 17:R257. [PubMed: 24168808]

40. Salluh JI, Wang H, Schneider EB, et al. Outcome of delirium in critically ill patients: systematic review and meta-analysis. BMJ. 2015; 350:h2538. [PubMed: 26041151]

41. Mathias JL, Wheaton P. Contribution of brain or biological reserve and cognitive or neural reserve to outcome after TBI: a meta-analysis (prior to 2015). Neurosci Biobehav Rev. 2015; 55:573-593. [PubMed: 26054792]

42. Roe CM, Xiong C, Miller JP, et al. Education and Alzheimer disease without dementia: support for the cognitive reserve hypothesis. Neurology. 2007; 68:223-228. [PubMed: 17224578]

43. Nouchi R, Taki Y, Takeuchi H, et al. Brain training game improves executive functions and processing speed in the elderly: a randomized controlled trial. PLoS One. 2012; 7:e29676. [PubMed: 22253758]

44. Gillis C, Li C, Lee L, et al. Prehabilitation versus rehabilitation: a randomized control trial in patients undergoing colorectal resection for cancer. Anesthesiology. 2014; 121:937-947. [PubMed: 25076007]

45. Patel SB, Poston JT, Pohlman A, et al. Rapidly reversible, sedation-related delirium versus persistent delirium in the intensive care unit. Am J Respir Crit Care Med. 2014; 189:658-665. [PubMed: 24423152] 

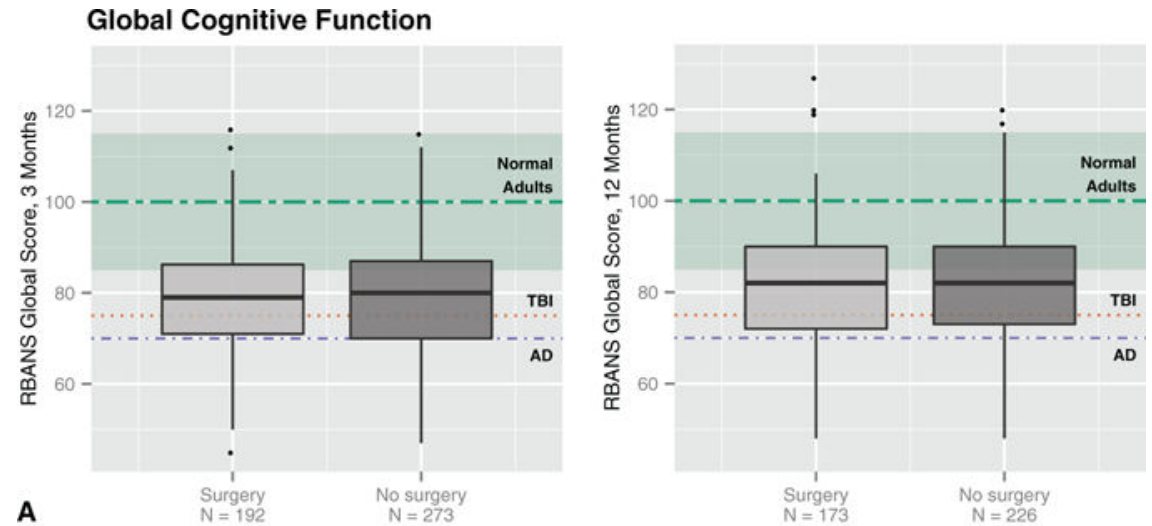

Executive Function
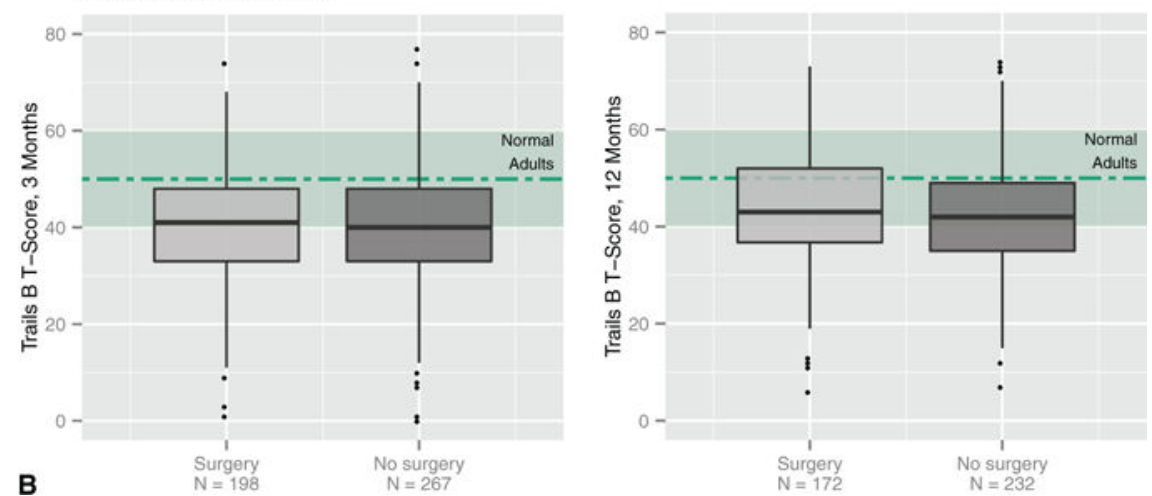

FIGURE 1.

Global cognition and executive function scores in patients with versus without surgery/ anesthesia exposure. The RBANS ${ }^{24}$ is a validated tool that examines global cognitive function and has a mean (standard deviation) population age-adjusted score of $100 \pm 15$ with lower scores indicating worse global cognitive function. The Trails $\mathrm{B}^{28}$ is a validated tool that examines executive function and has an age-, sex-, and education-adjusted mean score of $50 \pm 10$ with lower scores indicating worse executive function. RBANS scores were similar in patients with surgery/anesthesia exposure versus those without exposure, approximately 1.5 standard deviations below adjusted mean. Likewise, Trails B scores were similar in patients with surgery/anesthesia exposure versus those without exposure, approximately 1 standard deviation below the adjusted mean. The horizontal bar indicates the median, the upper and lower limits of the boxes indicate the interquartile range, the ends of the whiskers indicate 1.5 times the interquartile range, and the black dots indicate outliers. The green dashed line indicates the adjusted population mean for normal adults, and the green band indicates the standard deviation. Also shown are the expected RBANS mean scores for traumatic brain injury and Alzheimer disease from other cohort studies (patients $>65$ years of age in Alzheimer disease). 
TABLE 1

Demographic and Clinical Characteristics by Surgery Exposure

\begin{tabular}{|c|c|c|}
\hline Characteristics* & Surgery $(N=402)$ & No Surgery $(\mathrm{N}=638)$ \\
\hline Age at enrollment, yrs & $63(53-71)$ & $62(52-72)$ \\
\hline White race, $\mathrm{N}(\%)$ & $373(93 \%)$ & $573(90 \%)$ \\
\hline Male sex, $\mathrm{N}(\%)$ & $242(60 \%)$ & $385(60 \%)$ \\
\hline Education, yrs & $12(12-14)$ & $12(12-14)$ \\
\hline AHRQ Socioeconomic Status Index & $49.9(47.3-52.9)$ & $49.6(47.3-52.7)$ \\
\hline IQCODE-SF at enrollment & $3(3.00-3.12)$ & $3(3.00-3.12)$ \\
\hline \multicolumn{3}{|c|}{ Clinical Frailty Scale at enrollment, N (\%) } \\
\hline Very fit & $8(2 \%)$ & $23(4 \%)$ \\
\hline Well & $54(13 \%)$ & $79(12 \%)$ \\
\hline Well, treated comorbid disease & $146(36 \%)$ & $209(33 \%)$ \\
\hline Apparently vulnerable & $80(20 \%)$ & $134(21 \%)$ \\
\hline Mildly frail & $55(14 \%)$ & $85(13 \%)$ \\
\hline Moderately frail & $50(12 \%)$ & $85(13 \%)$ \\
\hline Severely frail & $9(2 \%)$ & $23(4 \%)$ \\
\hline \multicolumn{3}{|l|}{ FAQ score at enrollment, $\mathrm{N}(\%)$} \\
\hline No impairment (score 0-8) & $351(89 \%)$ & $541(87 \%)$ \\
\hline Some impairment (score $₫$ ) & $45(11 \%)$ & $80(13 \%)$ \\
\hline Charlson comorbidity index & $2.0(1.0-4.0)$ & $2.0(1.0-4.0)$ \\
\hline Framingham stroke risk & $10.0(6.0-14.0)$ & $10.0(6.0-15.0)$ \\
\hline SOFA score at enrollment & $9.0(7.0-12.0)$ & $9.0(7.0-11.0)$ \\
\hline APACHE II at admission & $25.0(19.0-31.0)$ & $23.0(17.2-29.0)$ \\
\hline Sepsis in the ICU, $\mathrm{N}(\%)$ & $272(68 \%)$ & $483(77 \%)$ \\
\hline Duration among exposed, $\mathrm{d}$ & $8.0(3.0-14.0)$ & $4.0(2.0-8.0)$ \\
\hline Mechanical ventilation, $\mathrm{N}(\%)$ & $384(96 \%)$ & $539(84 \%)$ \\
\hline Duration among exposed, $\mathrm{d}$ & $2.2(0.37-8.06)$ & $2.0(0.89-4.96)$ \\
\hline Delirium, N (\%) & $312(78 \%)$ & $428(67 \%)$ \\
\hline Duration among exposed, $\mathrm{d}$ & $5.0(2.0-10.0)$ & $3.0(2.0-6.0)$ \\
\hline Coma, N (\%) & $255(63 \%)$ & $372(58 \%)$ \\
\hline Duration among exposed, days & $4.0(2.0-7.0)$ & $3.0(1.0-5.0)$ \\
\hline \multicolumn{3}{|l|}{ Sedative or analgesic use, $\mathrm{N}(\%)$} \\
\hline Benzodiazepine & $279(69 \%)$ & $407(67 \%)$ \\
\hline Propofol & $222(55 \%)$ & $299(47 \%)$ \\
\hline Dexmedetomidine & $65(16 \%)$ & $63(10 \%)$ \\
\hline Opiate & $378(94 \%)$ & $456(71 \%)$ \\
\hline ICU length of stay, $d$ & $6.9(3.0-15.1)$ & $4.8(2.4-9.1)$ \\
\hline Hospital length of stay, $d$ & $12.8(7.1-22.0)$ & $8.8(5.2-14.1)$ \\
\hline In-hospital mortality, $\mathrm{N}(\%)$ & $60(15 \%)$ & $137(21 \%)$ \\
\hline One-year mortality, N (\%) & $149(37 \%)$ & $269(42 \%)$ \\
\hline
\end{tabular}

Ann Surg. Author manuscript; available in PMC 2018 March 16. 
AHRQ indicates Agency for Healthcare Research and Quality; APACHE, Acute Physiology and Chronic Health Evaluation; FAQ, Functional Activities Questionnaire; ICU, intensive care unit; IQCODE-SF, Short Form Informant Questionnaire on Cognitive Decline in the Elderly; SOFA, Sequential Organ Failure Assessment. 
TABLE 2

Effect of Surgery and Baseline Factors on Global Cognition and Executive Function Scores

\begin{tabular}{|c|c|c|c|c|}
\hline 3-Month Follow-Up (N = 494) & Adjusted RBANS Difference* & $P$ & Adjusted Trails B Difference* & $P$ \\
\hline Surgery/anesthesia exposure (yes/no) & $-1.35(-3.49$ to 0.79$)$ & 0.217 & $-0.07(-2.38$ to 2.24$)$ & 0.951 \\
\hline Age at enrollment, yrs & $-1.31(-4.79$ to 2.17$)$ & 0.001 & $-0.35(-2.28$ to 1.58$)$ & 0.724 \\
\hline Education, yrs & 4.78 (2.58 to 6.98$)$ & $<0.001$ & $0.17(-0.78$ to 1.12$)$ & 0.723 \\
\hline Charlson comorbidity index & $-2.58(-5.46$ to 0.31$)$ & 0.270 & $-1.39(-2.91$ to 0.13$)$ & 0.073 \\
\hline Framingham stroke risk & $0.60(-2.78$ to 3.98$)$ & 0.611 & $-1.03(-3.11$ to 1.05$)$ & 0.330 \\
\hline IQCODE-SF & $-1.19(-2.74$ to 0.36$)$ & $<0.001$ & $-0.59(-1.12$ to -0.06$)$ & 0.030 \\
\hline 12-Month Follow-Up ( $\mathrm{N}=413$ ) & Adjusted RBANS Difference* & $\boldsymbol{P}$ & Adjusted Trails B Difference* & $P$ \\
\hline Surgery/anesthesia exposure (yes/no) & $-0.99(-3.50$ to 1.52$)$ & 0.436 & $1.49(-0.90$ to 3.89$)$ & 0.220 \\
\hline Age at enrollment, yrs & $-0.90(-4.98$ to 3.18$)$ & 0.011 & $0.23(-1.80$ to 2.25$)$ & 0.826 \\
\hline Education, yrs & $4.50(1.99$ to 7.01$)$ & $<0.001$ & $0.40(-0.59$ to 1.40$)$ & 0.424 \\
\hline Charlson comorbidity index & $-0.66(-4.27$ to 2.94$)$ & 0.450 & $-0.33(-1.93$ to 1.27$)$ & 0.687 \\
\hline Framingham stroke risk & $0.41(-3.79$ to 4.61$)$ & 0.936 & $-1.48(-3.69$ to 0.73$)$ & 0.189 \\
\hline IQCODE-SF & 0.69 (-1.31 to 2.69$)$ & 0.038 & $-1.12(-1.64$ to -0.59$)$ & $<0.001$ \\
\hline
\end{tabular}

* A negative value is indicative of worse global cognition (RBANS) or executive function (Trails B) scores. The RBANS has a mean (standard deviation) population age-adjusted score of $100 \pm 15$; the Trails B has an age-, sex-, and education-adjusted mean score of $50 \pm 10$. We report effect size estimates with the adjusted outcome difference ( $95 \%$ confidence interval) from multivariable linear regression analyses for yes versus no for each dichotomous covariate and the cohort's 75 th versus 25 th percentile value for each continuous covariate. Continuous variables were modeled with the use of restricted cubic splines to allow for nonlinear associations between covariates and outcomes but require multiple beta coefficients to estimate the effect. The most appropriate $P$ value is one that takes into consideration all beta coefficients. Although the $P$ value may indicate significance (and is correct), the comparison of the 25 th and 75 th percentiles may yield a point estimate with a confidence interval that crosses zero, or vice versa.

IQCODE-SF indicates Short Form Informant Questionnaire on Cognitive Decline in the Elderly. 


\section{TABLE 3}

Effect of Surgery, Anesthesia, and Baseline Factors on Global Cognition and Executive Function Scores in a Subset of Surgical Patients

\begin{tabular}{|c|c|c|c|c|}
\hline 3-Month Follow-Up (N = 127) & Adjusted RBANS Difference* & $P$ & Adjusted Trails B Difference* & $P$ \\
\hline Number of surgeries & $1.22(-3.93$ to 6.38$)$ & 0.285 & $-2.15(-5.09$ to 0.80$)$ & 0.151 \\
\hline Emergent surgery (yes/no) & $2.47(-1.98$ to 6.93$)$ & 0.274 & $-1.60(-7.10$ to 3.89$)$ & 0.565 \\
\hline Total anesthesia duration, min & $-0.01(-3.96$ to 3.93$)$ & 0.190 & $1.02(-2.84$ to 4.87$)$ & 0.603 \\
\hline Age at enrollment, yrs & $-1.41(-4.16$ to 1.35$)$ & 0.081 & $-1.57(-4.86$ to 1.73$)$ & 0.348 \\
\hline Education, yrs & 3.97 (2.09 to 5.84$)$ & $<0.001$ & $1.02(-0.99$ to 3.04$)$ & 0.317 \\
\hline Charlson comorbidity index & $-0.53(-3.85$ to 2.80$)$ & 0.889 & $-1.78(-5.53$ to 1.97$)$ & 0.348 \\
\hline IQCODE-SF & $-2.28(-4.92$ to 0.35$)$ & $<0.001$ & $-0.58(-1.20$ to 0.05$)$ & 0.071 \\
\hline 12-Month Follow-Up ( $=111)$ & Adjusted RBANS Difference* & $P$ & Adjusted Trails B Difference* & $P$ \\
\hline Number of surgeries & $1.67(-2.95$ to 6.29$)$ & 0.072 & $-0.66(-4.04$ to 2.73$)$ & 0.702 \\
\hline Emergent surgery (yes/no) & $3.47(-1.82$ to 8.76$)$ & 0.196 & $3.05(-2.71$ to 8.80$)$ & 0.296 \\
\hline Total anesthesia duration, min & $-4.71(-8.83$ to -0.59$)$ & 0.066 & $-1.75(-5.43$ to 1.93$)$ & 0.347 \\
\hline Age at enrollment, yrs & $-0.08(-3.57$ to 3.41$)$ & 0.125 & -0.86 ( -4.22 to 2.50$)$ & 0.614 \\
\hline Education, yrs & 3.94 (1.63 to 6.24$)$ & $<0.001$ & $0.50(-1.48$ to 2.47$)$ & 0.618 \\
\hline Charlson comorbidity index & -0.33 (-3.89 to 3.23$)$ & 0.963 & -0.04 (-3.61 to 3.52$)$ & 0.980 \\
\hline IQCODE-SF & $-1.41(-4.97$ to 2.15$)$ & 0.060 & $-0.99(-1.61$ to -0.37$)$ & 0.002 \\
\hline
\end{tabular}

* A negative value is indicative of worse global cognition (RBANS) or executive function (Trails B) scores. The RBANS has a mean (standard deviation) population age-adjusted score of $100 \pm 15$; the Trails B has an age-, sex-, and education-adjusted mean score of $50 \pm 10$. We report effect size estimates with the adjusted outcome difference ( $95 \%$ confidence interval) from multivariable linear regression analyses for yes versus no for each dichotomous covariate and the cohort's 75 th versus 25 th percentile value for each continuous covariate. Continuous variables were modeled with the use of restricted cubic splines to allow for nonlinear associations between covariates and outcomes but require multiple beta coefficients to estimate the effect. The most appropriate $P$ value is one that takes into consideration all beta coefficients. Although the $P$ value may indicate significance (and is correct), the comparison of the 25 th and 75 th percentiles may yield a point estimate with a confidence interval that crosses zero, or vice versa.

IQCODE-SF indicates Short Form Informant Questionnaire on Cognitive Decline in the Elderly. 
TABLE 4

Effect of Surgery, Baseline, and In-Hospital Factors on Global Cognition and Executive Function Scores

\begin{tabular}{|c|c|c|c|c|}
\hline 3-Month Follow-Up (N = 494) & Adjusted RBANS Difference* & $P$ & Adjusted Trails B Difference* & $P$ \\
\hline Surgery/anesthesia exposure (yes/no) & $-1.04(-3.48$ to 1.40$)$ & 0.404 & 0.42 ( -2.17 to 3.02$)$ & 0.749 \\
\hline Age at enrollment, yrs & $-0.91(-4.58$ to 2.76$)$ & $<0.001$ & $0.63(-3.25$ to 4.51$)$ & 0.230 \\
\hline Education, yrs & $4.67(2.43$ to 6.90$)$ & $<0.001$ & $-0.25(-2.65$ to 2.14$)$ & 0.894 \\
\hline Charlson comorbidity index & $-2.52(-5.57$ to 0.54$)$ & 0.274 & $-3.77(-7.10$ to -0.43$)$ & 0.026 \\
\hline Framingham stroke risk & $1.05(-2.44$ to 4.54$)$ & 0.547 & $1.37(-2.32$ to 5.06$)$ & 0.391 \\
\hline IQCODE-SF & $-0.81(-2.39$ to 0.76$)$ & $<0.001$ & $0.82(-0.86$ to 2.49$)$ & 0.100 \\
\hline Delirium duration, $\mathrm{d}$ & $-5.43(-8.82$ to -2.03$)$ & 0.012 & $-3.55(-7.84$ to 0.74$)$ & 0.010 \\
\hline Coma duration, $\mathrm{d}$ & $-1.47(-4.73$ to 1.78$)$ & 0.537 & $2.16(-3.36$ to 7.68$)$ & 0.148 \\
\hline Severe sepsis duration, $\mathrm{d}$ & $2.10(-0.84$ to 5.03$)$ & 0.372 & 1.13 ( -2.07 to 4.34$)$ & 0.767 \\
\hline Hypoxemia, 15 min intervals & $0.70(-1.84$ to 3.23$)$ & 0.250 & $-2.11(-4.85$ to 0.62$)$ & 0.321 \\
\hline Mean SOFA score & $1.15(-1.83$ to 4.14$)$ & 0.839 & $0.85(-2.33$ to 4.03$)$ & 0.886 \\
\hline Mean benzodiazepines ${ }^{\dagger}$ & $0.57(-2.55$ to 3.69$)$ & 0.564 & $-3.03(-6.40$ to 0.34$)$ & 0.176 \\
\hline Mean propofol ${ }^{\dagger}$ & $-0.65(-3.60$ to 2.29$)$ & 0.923 & $-3.31(-6.42$ to -0.21$)$ & 0.214 \\
\hline Mean dexmedetomidine ${ }^{\dagger}$ & $-0.70(-7.23$ to 5.83$)$ & 0.433 & $-1.24(-8.31$ to 5.83$)$ & 0.731 \\
\hline Mean opiates ${ }^{\dagger}$ & $2.60(-0.97$ to 6.17$)$ & 0.145 & $5.66(1.79$ to 9.54$)$ & 0.039 \\
\hline Mean haloperidol ${ }^{\dagger}$ & $-2.44(-8.54$ to 3.66$)$ & 0.433 & $-0.33(-6.22$ to 6.89$)$ & 0.921 \\
\hline 12-Month Follow-Up ( $=413)$ & Adjusted RBANS Difference* & $P$ & Adjusted Trails B Difference* & $P$ \\
\hline Surgery/anesthesia exposure (yes/no) & $-0.47(-3.28$ to 2.33$)$ & 0.741 & $1.35(-1.23$ to 3.93$)$ & 0.305 \\
\hline Age at enrollment, yrs & $-2.38(-6.67$ to 1.91$)$ & 0.012 & $0.87(-1.24$ to 2.99$)$ & 0.417 \\
\hline Education, yrs & 4.01 (1.48 to 6.54$)$ & $<0.001$ & $0.46(-0.54$ to 1.46$)$ & 0.364 \\
\hline Charlson comorbidity index & $0.66(-3.12$ to 4.44$)$ & 0.151 & $-0.54(-2.19$ to 1.11$)$ & 0.519 \\
\hline Framingham stroke risk & $1.05(-3.26$ to 5.35$)$ & 0.888 & $-0.97(-3.22$ to 1.27$)$ & 0.393 \\
\hline IQCODE-SF & $1.15(-0.85$ to 3.16$)$ & 0.073 & $-1.10(-1.63$ to -0.58$)$ & $<0.001$ \\
\hline Delirium duration, $\mathrm{d}$ & $-7.28(-11.35$ to -3.22$)$ & 0.003 & $-2.96(-4.70$ to -1.22$)$ & $<0.001$ \\
\hline Coma duration, $\mathrm{d}$ & $0.37(-3.50$ to 4.24$)$ & 0.518 & 0.34 (-1.27 to 1.96$)$ & 0.679 \\
\hline Severe sepsis duration, $\mathrm{d}$ & $0.78(-2.77$ to 4.32$)$ & 0.726 & $0.71(-0.86$ to 2.28$)$ & 0.374 \\
\hline Hypoxemia, 15 min intervals & $2.06(0.39$ to 3.73$)$ & 0.040 & $-0.09(-0.40$ to 0.21$)$ & 0.542 \\
\hline Mean SOFA score & $-0.97(-4.45$ to 2.52$)$ & 0.587 & $0.70(-1.04$ to 2.44$)$ & 0.432 \\
\hline Mean benzodiazepines ${ }^{\dagger}$ & $-0.59(-4.27$ to 3.10$)$ & 0.178 & $1.03(-1.31$ to 3.36$)$ & 0.387 \\
\hline Mean propofol ${ }^{\dagger}$ & $-1.40(-4.69$ to 1.88$)$ & 0.666 & $-1.18(-3.23$ to 0.87$)$ & 0.259 \\
\hline Mean dexmedetomidine ${ }^{\dagger}$ & $-4.38(-12.08$ to 3.32$)$ & 0.264 & $-2.43(-9.44$ to 4.59$)$ & 0.497 \\
\hline Mean opiates ${ }^{\dagger}$ & $0.87(-3.36$ to 5.09$)$ & 0.036 & $1.88(-0.65$ to 4.41$)$ & 0.145 \\
\hline Mean haloperidol ${ }^{\dagger}$ & $-3.28(-10.21$ to 3.65$)$ & 0.352 & $-0.64(-7.26$ to 5.99$)$ & 0.850 \\
\hline
\end{tabular}

* A negative value is indicative of worse global cognition (RBANS) or executive function (Trails B) scores. The RBANS has a mean (standard deviation) population age-adjusted score of $100 \pm 15$; the Trails B has an age-, sex-, and education-adjusted mean score of $50 \pm 10$. We report effect size estimates with the adjusted outcome difference ( $95 \%$ confidence interval) from multivariable linear regression analyses for yes versus no for each dichotomous covariate and the cohort's 75 th versus 25 th percentile value for each continuous covariate. Continuous variables were modeled with the use of restricted cubic splines to allow for nonlinear associations between covariates and outcomes but require multiple beta coefficients to 
estimate the effect. The most appropriate $P$ value is one that takes into consideration all beta coefficients. Although the $P$ value may indicate significance (and is correct), the comparison of the 25 th and 75 th percentiles may yield a point estimate with a confidence interval that crosses zero, or vice versa.

${ }^{\dagger}$ Mean 24-hour doses on days exposed, cube root transformed to minimize impact of outlier doses.

IQCODE-SF indicates Short-Form Informant Questionnaire on Cognitive Decline in the Elderly; SOFA, Sequential Organ Failure Assessment. 